\title{
Stream Distributed Coded Computing
}

\author{
Alejandro Cohen*, Guillaume Thiran ${ }^{\dagger}$, Homa Esfahanizadeh*, and Muriel Médard* \\ *RLE, MIT, Cambridge, MA, USA, \{cohenale, homaesf, medard\} @ mit.edu \\ ${ }^{\dagger}$ UCLouvain, Belgium, guillaume.thiran@uclouvain.be
}

\begin{abstract}
The emerging large-scale and data-hungry algorithms require the computations to be delegated from a central server to several worker nodes. One major challenge in the distributed computations is to tackle delays and failures caused by the stragglers. To address this challenge, introducing efficient amount of redundant computations via distributed coded computation has received significant attention. Recent approaches in this area have mainly focused on introducing minimum computational redundancies to tolerate certain number of stragglers. To the best of our knowledge, the current literature lacks a unified end-to-end design in a heterogeneous setting where the workers can vary in their computation and communication capabilities. The contribution of this paper is to devise a novel framework for joint scheduling-coding, in a setting where the workers and the arrival of stream computational jobs are based on stochastic models. In our initial joint scheme, we propose a systematic framework that illustrates how to select a set of workers and how to split the computational load among the selected workers based on their differences in order to minimize the average in-order job execution delay. Through simulations, we demonstrate that the performance of our framework is dramatically better than the performance of naive method that splits the computational load uniformly among the workers, and it is close to the ideal performance.
\end{abstract}

\section{Index Terms}

Distributed coded computation, stragglers, large matrix-matrix multiplication, large matrix-vector multiplication, ultra-reliable low-latency, in-order execution delay, queuing theory.

\section{INTRODUCTION}

We are in the era of big data where the size and dimension of data grow dramatically surpassing the Moore's law [1], [2]. As a result, the central heavy computations over massive amount of data have become more unrealistic, and distributing the computations over several workers by a master node has become the rising solution [3]-[5] computational job to be split into several tasks with lower complexity and smaller input data, such that they can be distributed among a set of workers and the final job result can be obtained

Authors have equal contributions.

${ }^{1}$ In another view of distributed computation, which is not the focus of this paper, there is no master to orchestrate, and the workers coordinate among themselves. 


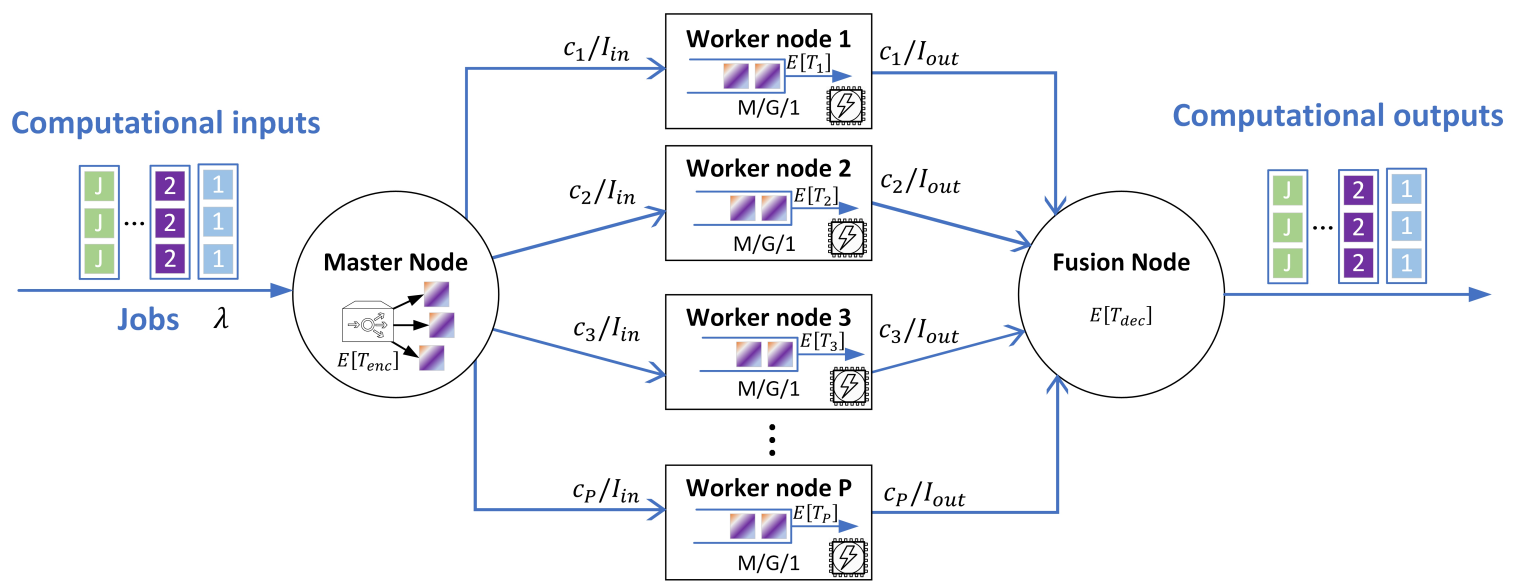

Fig. 1: System model for the distributed computation problem. The parameters are: $\lambda$ for the average job arrival rate. $E\left[T_{\text {enc }}\right]$ and $E\left[T_{\text {dec }}\right]$ for the average encoding time and decoding time, respectively. $E\left[T_{p}\right], c_{p} / I_{\text {in }}$, and $c_{p} / I_{\text {out }}$ for the average computing time, incoming communication time, and outgoing communication time per job, respectively. Each worker is modeled with an M/G/1 queue.

by a fusion node using the combined task results. Fig. 1 illustrates a system model for the distributed computation problem.

One major challenge in the distributed computation framework is addressing delays and failures caused by slow and/or unreliable workers known as stragglers affecting the overall performance of the system [6]-[8]. The efforts to reduce the bottleneck effect of stragglers has opened up a new line of research as distributed coded computation that has attracted significant attention [9]-[11]. The idea is to introduce redundancies in the distributed computations such that the output can be retrieved from an arbitrary subset of task results, and so the performance of the system will not be limited by the stragglers [12], [13].

The majority of previous works on distributed coded computation focus on decoding the result of a computational job given a subset of successful task results assuming a homogeneous cluster of workers [4]. In practice, there is a variance among computational powers of the workers depending on their CPU/GPU powers, aging factors, maintenance conditions, etc [14], [15]. Besides, the communication links to and from each worker can have various rates causing various communication latency [4], [16], [17]. Incorporating the heterogeneity of the workers into the distributed coded computation design has been less studied in the literature. In [18], a model is considered where maximum-distance-separable (MDS) codes [19]-[22] are used for generating computational redundancies, and redundant computations are removed from queue of workers once enough tasks are finished. It is shown via a precise queuing analysis that replication codes result in a higher delay compared to the MDS codes in this setting. However, a scheduling that incorporates the variabilities at the workers into the design is an open challenge. 


\section{Main Contribution}

In this paper, we propose a new adaptive framework to execute a stream of non-iterative computational jobs with low in-order execution delay in a distributed fashion, see Fig. 1. We consider each worker is modeled with an M/G/1 queue ${ }^{2}$, and the workers can vary in their service rates. This is a first step to design a joint scheduling-coding framework for stream distributed computation utilizing queuing preliminaries. The average job execution delay depends on the system model parameters, parameters of the coding scheme for splitting the jobs into smaller tasks, and scheduling scheme for distribution of the tasks among the acquired workers. The scheduling-coding optimization is critical to minimize the in-order execution delay of the jobs and to maximize the utilization of the workers.

To this end, the proposed framework selects optimal code parameters and optimally splits the computations among the workers based on their computational and communication power. Despite the previous works which focus on one or few targets, we consider all sources of delays in our end-to-end system design, i.e., encoding/decoding delay, queuing delay, computation delay, and communication delay, to optimize the parameters. Our solution provides a balance between the end-to-end in-order execution delay and the total acquired computational resources for stream computing applications. Our framework is compatible with a general form of distributed computation and can be combined with many existing coded computation codes, e.g., [3], [9], [23]-[26], to optimize their code parameters in order to reduce the in-order execution delay and improve their utilization.

Our method can be briefly described as follows. We assume a possibly large set of workers are available to select from. We first choose an appropriate set of workers that provides sufficient resources and keeps the system stable. Then, the optimal load split (contribution portion of each worker) is identified to minimize the in-order execution delay. This optimization process is repeated periodically during the run-time, or when changes occur in the system ${ }^{3}$. We note that the optimal load split depends on first and second moment of each worker's service rate, and can be obtained via feedback and/or via prior knowledge of the master node.

The rest of this paper is organized as follows. In Section $\Pi$, we summarize the related works. In Section III, we describe the system model. In Section IV, we propose our new framework for joint scheduling-coding in a distributed computation problem. In Section V, we describe how to adaptively adjust the parameters needed for our proposed optimal solution using feedback. Sections $\mathrm{VI}$ and $\mathrm{VII}$ are dedicated to simulation results and conclusions, respectively.

\footnotetext{
${ }^{2}$ In queuing theory, an M/G/1 queue is a model with a single processor where arrivals have Poisson distribution and service times have a General distribution.

${ }^{3}$ The memory-less job arrival model at the workers, i.e., M/G/1 model, is a result of the memory-less job arrival model at the master node. This model leads us to provide an optimal load split using the various stochastic features of the service time at the workers. Considering a general job arrival distribution at the master node requires more complex considerations in order to formulate the average job execution delay of the system. We leave this interesting extension as future work.
} 


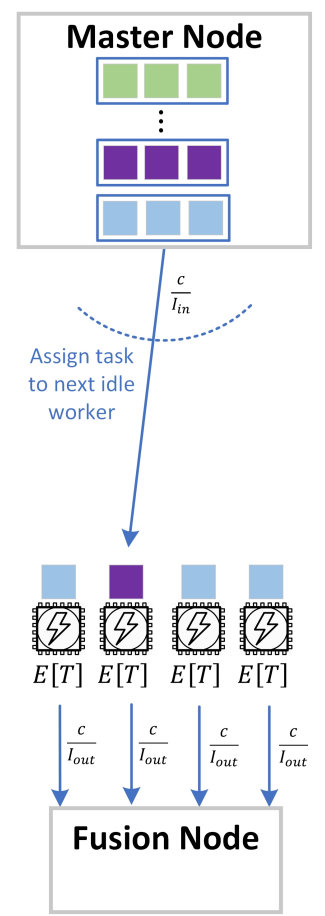

(a) Ideal

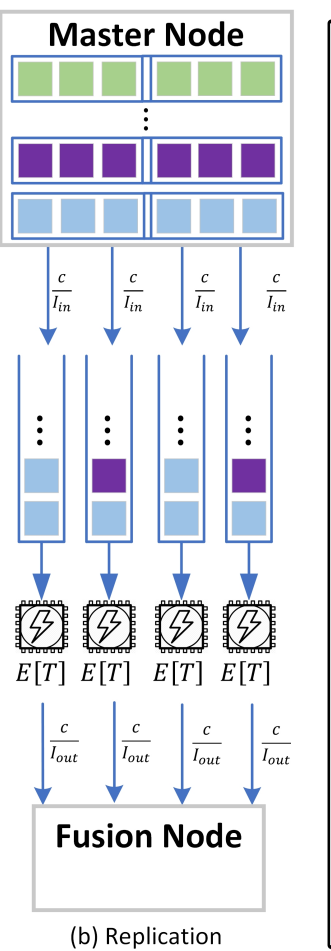

(b) Replication

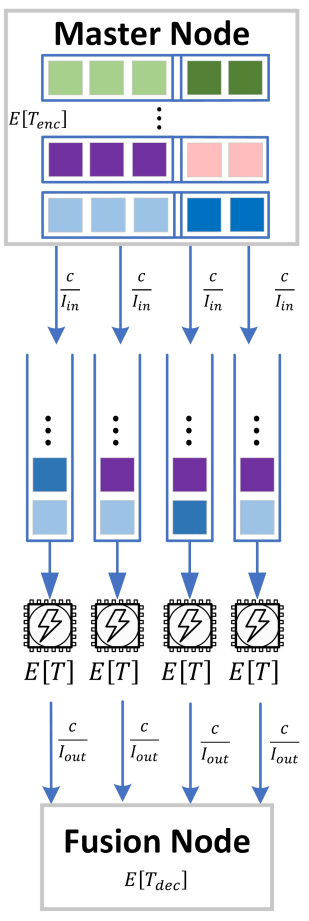

(c) MDS/Rateless Coded

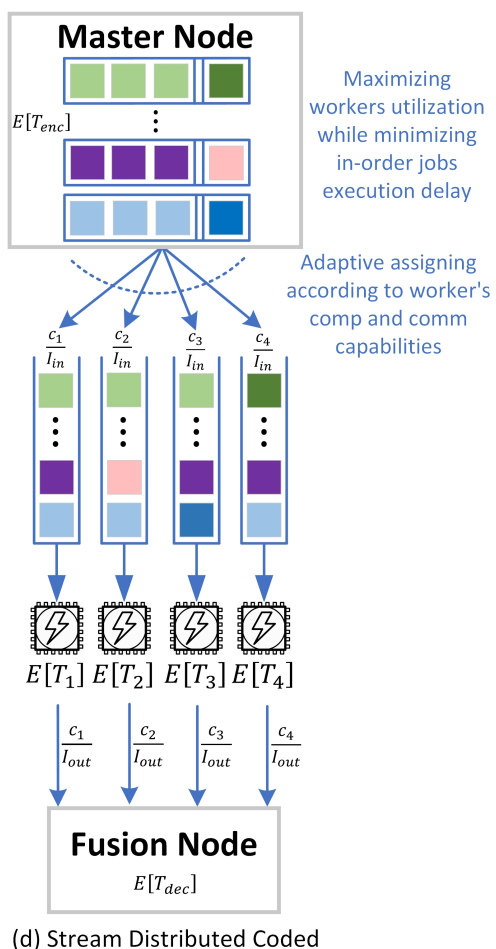

(d) Stream Distributed Coded

Fig. 2: Various distributed computational schemes. Each computational job is split to several smaller tasks. The tasks related to one job are marked with the same color, and the redundant computations are distinguished from the necessary ones with a lighter color.

\section{RELATED WORK}

How to introduce computational redundancies in distributed computation has been considered in many modern applications that require heavy computations over a large amount of data. An interesting example is multiplication of two large matrices [19], [20], [22], [26]-[28] and multiplication of a large matrix and a vector [3], [4]. Another example is MapReduce where a computationally intensive job of identifying specific features over a possibly large dataset, e.g., specific word counts in a large file, is distributed among several workers such that each worker performs the process over one or several smaller subsets of the dataset [29]. Random shuffling of data is another attractive example which benefits from the coded computation and enhances the performance of large-scale machine learning algorithms [30], [31]. There, work mainly focuses on optimizing the code parameters to pursue goals such as reducing the number of task results required to finish a job [26], [27], providing a balance between computation load and communication load [19], reducing the encoding and decoding complexities [28], improving the communication efficiency [30], [31], among others. Recently, an information theoretic model of functional compression for distributed computation has been studied in [17].

Fig. 2 depicts various scheduling-coding methods for the distributed coded computation problem. (a) is the ideal solution, where in-order the jobs received at the master node are assigned 
to the workers as soon as they become idle. (b) is the replication solution, where the tasks for each job are replicated and then uniformly distributed among the workers. (c) is coding solution, where the tasks, being the result of a distributed coding scheme, eg., MDS codes [19], [20] and rateless codes [4] and including some redundant tasks, are generated and assigned to the workers. (d) is our proposed joint coding and scheduling framework that incorporates the diversities of the workers to optimally split the task load of each job among the workers. According to our simulation results, the optimal distribution of computational jobs according to our framework not only results in an average in-order execution delay much lower than the uniform job distribution, but it also is capable to provide stability in cases that the uniform split is not able to do so.

\section{SYSTEM MODEL}

Fig. 1] illustrates the setting for distributed computational of a stream of computational jobs with latency constraints. The master node sequentially receives the inputs for several computational jobs. We model the job arrival at the master with a Poisson stochastic random process $\mathcal{N}(l)$ with parameter $\lambda$ that represents the number of jobs arriving up to and including time step $l, l \in\{1, \ldots, L\}$,

$$
P(\mathcal{N}(l)=n)=e^{-\lambda l} \frac{(\lambda l)^{n}}{n !} .
$$

Thus, $\lambda$ also denotes the average job arrival rate at each time step, and we assume $J=\mathcal{N}(L)$.

Upon arrival of each job, the master node assigns appropriate portion of the computational job, i.e., appropriate number of tasks, to each worker in a selected set of workers $\mathcal{P}$, where $|\mathcal{P}|=P$. The queue at each worker is modeled with an M/G/1 queue [32]. Let $\phi_{p}$ be the portion of a job that is assigned to the $p$-th worker (which will be designed by our solution), $p \in\{1, \ldots, P\}$. These $P$ workers are selected from a large set of workers by the proposed framework. Each worker independently performs pre-defined computational operations and sends the results to a fusion node where the received computational results from workers are aggregated to produce the desired computational output for each job, in-order. We denote as $I_{\mathrm{in}}, I_{\text {out }}$, and $C$ the input size (number of symbols), output size (number of symbols), and computational complexity (number of operations) for each job.

Each worker has limited computational resources and communication capacity at each time step. That is, each worker can perform operations on and transmit a limited amount of computational inputs at a time. Besides, the computational speed and communication rate can vary from worker to worker. We denote as $c_{p}$ the transmission rate of the $p$-th worker, i.e., the number of symbols that can be communicated to or from the $p$-th worker at each time step. Let $T_{p}$ denote the time it takes for $p$-th worker to perform one entire computational job, i.e., $C$ operations. Then $E\left[T_{p}\right]$ and $E\left[T_{p}^{2}\right]$ denote the first moment (average) and the second moment of the service time for an entire job for the $p$-th worker, respectively, and the service rate of the worker for entire job is $1 / E\left[T_{p}\right]$. The master node has access to $E\left[T_{p}\right]$ and $E\left[T_{p}^{2}\right]$ according to the workers' 
declaration and/or estimations during the execution, which will be explained thouroughly in Section $\mathrm{V}$-A

We assume the first moment and second moment of the time it takes for the $p$-th worker to finish $\phi_{p}$ portion of a job is $\phi_{p} E\left[T_{p}\right]$ and $\phi_{p}^{2} E\left[T_{p}^{2}\right]$, respectively. We define the scaled service rate

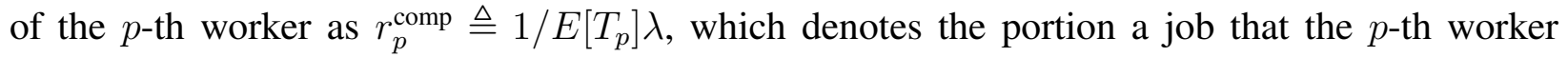
is able to perform on average during $1 / \lambda$ time steps. We also define the scaled communication rate of the $p$-th worker as $r_{p}^{\text {comm }} \triangleq c_{p} /\left(I_{\text {in }}+I_{\text {out }}\right) \lambda$, which denotes the portion a job that the $p$-th worker is able to transfer during $1 / \lambda$ time steps. We remind that $\phi_{p}$ is the portion of one job that is assigned to the $p$-th worker by the designed solution and $r_{p}^{\text {comp }}$ is the portion one job that the $p$-th worker is able to perform on average during $1 / \lambda$ time steps according to its declaration and/or the master node's estimation. Then, the average time it takes for the system to compute a job is $\frac{1}{P} \sum_{p=1}^{P} \phi_{p} E\left[T_{p}\right]$, and

$$
\frac{1}{P} \sum_{p=1}^{P} \phi_{p} E\left[T_{p}\right] \leq \frac{1}{P} \sum_{p=1}^{P} r_{p}^{\mathrm{comp}} E\left[T_{p}\right],
$$

where we require for stability that the time it takes to solve a job is lower than the inverse of the arrival rate. Now, we consider the formulation of the computational jobs. Let consider the $j$-th computational job, $j \in\{1, \ldots, J\}$, arrived at the master node as

$$
f\left(X_{1}(j), X_{2}(j), \ldots, X_{m}(j)\right)
$$

where $X_{1}(j), X_{2}(j), \ldots, X_{m}(j)$ are the computational inputs for the $j$-th job, and $f($.$) describes$ the computational job. Each job can be divided into several smaller computational tasks that has smaller inputs and lower computational complexity. Each computational task works toward completion of a single job, the computational tasks work on inputs of the same size, and the input of a computational task does not have to be one of the computational inputs (it can be a function of them). We assume each task can be performed by one worker and requires a specific number of operations. The fusion node requires to obtain $K$ completed task results, each with the same size, per job to identify the final result.

In this paper, as representative example of computational jobs, we consider the distributed multiplication of two large matrices using PolyDot scheme [26]. This coding scheme will be reviewed in more detail in Appendix $\mathrm{A}$. The reason we choose Polydot scheme for distributed matrix multiplication in this paper is the flexibility it offers to balance the computational complexity (minimum required workers) and computational traffic per worker. This flexibility can be utilized later, via some hyper parameters, in designing the bigger solution in order to obtain the desired trade-off among the system performance metrics. We note that the proposed stream computing solution is compatible with any distributed computational job in form of (1), e.g., Polynomial codes, Matdots codes, $d$-dimensional product codes [33], Short-Dot codes [3], and 


\begin{tabular}{|l|l|}
\hline Parameter & Definition \\
\hline \hline$l$ & time step index, $l \in\{1, \ldots, L\}$ \\
\hline$\lambda$ & average job arrival rate \\
\hline $\mathcal{P}$ and $P$ & set of acquired workers and its cardinality \\
\hline$T_{p}$ & job service time of the $p$-th worker \\
\hline$r_{p}^{\text {comp }}$ & scaled service rate of the $p$-th worker \\
\hline$r_{p}^{\text {comm }}$ & scaled communication rate of the $p$-th worker \\
\hline$\phi_{p}$ & fraction of load on the $p$-th worker \\
\hline$\mu_{p}$ & computing rate of operations for the $p$-th worker \\
\hline$K$ & number of critical tasks per job \\
\hline$\Omega$ & redundancy ratio \\
\hline
\end{tabular}

TABLE I: Table of frequently visited parameters.

many others. Table $[$ shows a list of frequently visited parameters throughout this paper along with their short definitions.

\section{Scheduling and Failure Control Solution for Stream Coded Computing}

The goal of this section is proposing a systematic framework to identify the parameters of the system, i.e., selecting the set of workers and splitting the computational load among them, such that a stable computational solution with high throughput (high utilization of the acquired workers) and low in-order execution delay (in-order delivery delay of the jobs at the fusion node) is obtained. Our proposed solution efficiently combines the distributed computation idea with scheduling mechanism to improve the throughput and lower the in-order execution delay.

When a job arrives at the master node, the master node selects an appropriate set of workers and distribute the computational load among them. The first $K$ tasks related to a job (the minimum number of tasks for completion of a job) are called critical tasks 4 . The subsequent tasks related to a job are called supportive tasks, which compensate for the system failures. Later in Section $\mathrm{V}-\mathrm{B}$, we introduce a variant of the solution that tracks the real-time realization of errors and delays during one job service time to further improve the performance by assigning feed-back based supportive tasks.

Definition 1 (Task generation rate). Task generation rate of the system is defined as $K \Omega$, which denotes the number tasks generated per job. The parameter $\Omega \geq 1$ is called redundancy ratio.

The $(\Omega-1) K$ supportive tasks per job compensate for the system stragglers, i.e., the workers that take much longer than expected to finish an assigned task. Thus, $\Omega$ provides a trade-off between the system throughput and delay such that larger $\Omega$ results in more redundancy (lower throughput) and also lower delay.

\footnotetext{
${ }^{4}$ Since in our model, we may assign multiple tasks per job to a worker, the condition $K \leq P$ is not necessary.
} 
Assumption 1. For each worker with index $p, p \in\{1, \ldots P\}$, the incoming traffic rate $c_{p} / I_{\text {in }}$, the outgoing traffic rate $c_{p} / I_{\text {out }}$, the encoding rate of the master node, and the decoding rate of the fusion node are all equal to or larger than the average job service rate $1 / E\left[T_{p}\right]$. Thus, the encoding, decoding, and communication are not sources of a bottleneck for the workers.

We note that if Assumption 1 does not hold an extra delay is introduced to the system that needs to be incorporated in the analysis. In case the communication time from the master node to each worker is negligible, i.e., $c_{p} \rightarrow \infty$, the workers can use the master node buffer.

Definition 2 (Valid Worker). A worker is valid for a distributed coded computation if its parameters satisfy Assumption 1. In other words,

$$
\min \left(\frac{1}{E\left[T_{\text {enc }}\right]}, \frac{c_{p}}{I_{\text {in }}}, \frac{c_{p}}{I_{\text {out }}}, \frac{1}{E\left[T_{\text {dec }}\right]}\right) \geq \frac{1}{E\left[T_{p}\right]}
$$

Here, $E\left[T_{\mathrm{enc}}\right]$ is the average encoding time (for generation of $K \Omega$ ) at the master node, and $E\left[T_{\mathrm{dec}}\right]$ is the average decoding time per job at the fusion node (given $K$ successful task results).

Assumption 2. For a stable system,

$$
\lambda \leq \sum_{p=1}^{P} 1 / E\left[T_{p}\right] .
$$

Moreover, since $\phi_{p}$ portion of a job is assigned to the $p$-th worker, $p \in\{1, \ldots, P\}$, on average one job is assigned to the $p$-th worker during $\lambda \phi_{p}$ time steps. For stability of each worker, this time must be equal or greater then the average time it takes for the $p$-th worker to perform one job, i.e., $E\left[T_{p}\right]$, resulting in the following stronger stability condition.

Assumption 3. For stability at the workers,

$$
\phi_{p} \leq r_{p}^{\text {comp }}, \quad \forall p \in\{1, \ldots, P\}
$$

Since $\sum_{p=1}^{P} \phi_{p}=1$ in the proposed parallel setting, Assumption 3 guarantees Assumption 2 . and it needs to hold for any stable solution to avoid queues' overflows.

For a choice of hyper parameters for the distributed coded computation scheme, e.g., $s$ and $t$ for the PolyDot scheme, a set of valid codes that satisfy Assumption 2 are selected. The optimal load split among the selected workers, i.e., $\left\{\phi_{1}, \ldots, \phi_{P}\right\}$, is determined to minimize the average job execution time under Assumption 3. The best choice for hyper parameters of the computation scheme along with the corresponding set of workers and the optimal load split among them are recorded for utilization. The procedure is repeated periodically or whenever changes occur in the system. 


\section{A. Optimized Split of Computational Load}

Since the problem setting is heterogeneous and workers have various computational powers and transmission rates, the computational load at the master node needs to be split appropriately among the workers. The parameter $\phi_{p} \in[0,1]$ denotes the fraction of computational load on the $p$-th worker, such that $\sum_{p=1}^{P} \phi_{p}=1$. Considering the M/G/1 queuing model of the workers, the job arrival rate is $\lambda \phi_{p}$, the first and second moments of the job service time are $E\left[T_{p}\right]$ and $E\left[T_{p}^{2}\right]$, respectively, and the work load at the queue at queue of the $p$-th worker is $\rho_{p}=\lambda \phi_{p} E\left[T_{p}\right]$. Hence the average time it takes for the $p$-thworker to respond to a job, including both waiting time in the queue and the processing time, is given by Pollaczek-Khinchin formula [34, Chapter 5],

$$
D_{\text {comp }, p}=\frac{\lambda \phi_{p} E\left[T_{p}^{2}\right]}{2\left(1-\rho_{p}\right)}+E\left[T_{p}\right]=0.5 \frac{E\left[T_{p}^{2}\right]}{E\left[T_{p}\right]} \frac{\phi_{p}}{\frac{1}{\lambda E\left[T_{p}\right]}-\phi_{p}}+E\left[T_{p}\right]=\frac{1}{\lambda}\left(\frac{a_{p} \phi_{p}}{r_{p}^{\text {comp }}-\phi_{p}}+\frac{1}{r_{p}^{\text {comp }}}\right) .
$$

We remind that $r_{p}^{\text {comp }}=1 / \lambda E\left[T_{p}\right]$ and $a_{p} \triangleq 0.5 \lambda E\left[T_{p}^{2}\right] / E\left[T_{p}\right]$.

Definition 3 (Average job computation time). The average job computation time is defined as the average response time to a job averaged over all workers and normalized by $P$ (because of the parallel setting),

$$
D_{\text {comp }}=\frac{1}{P} \sum_{p=1}^{P} \frac{1}{\lambda}\left(\frac{a_{p} \phi_{p}^{2}}{r_{p}^{c o m p}-\phi_{p}}+\frac{\phi_{p}}{r_{p}^{\text {comp }}}\right) .
$$

We remind that $\lambda r_{p}^{\text {comm }}=c_{p} /\left(I_{\text {in }}+I_{\text {out }}\right)$ is the average job transmission rate for the $p$-th worker, $p \in\{1, \ldots, P\}$, including both communication from master node to the worker and from the worker to the fusion node.

Definition 4 (Average job communication time). It is defined as the average job transmission time averaged over all workers and normalized by $P$ (because of the parallel setting),

$$
D_{\text {comm }}=\frac{1}{P} \sum_{p=1}^{P} \frac{\phi_{p}}{\lambda r_{p}^{c o m m}}
$$

We remind that $E\left[T_{\text {enc }}\right]$ and $E\left[T_{\mathrm{dec}}\right]$ denote the average encoding time per job, i.e., generating $K \Omega$ tasks at the master node, and decoding time per job, i.e., decoding the final job result given receiving $K$ successful task results, respectively.

Definition 5 (Average job execution time). The average job execution time is given by

$$
D_{\text {exe }}=D_{\text {comp }}+D_{\text {comm }}+E\left[T_{\text {enc }}\right]+E\left[T_{\text {enc }}\right]
$$

As the workers are heterogeneous, the way the computational load is split among them must be optimized to minimize the average job execution times. The problem of optimal load split is

\footnotetext{
${ }^{5} \mathrm{We}$ do not consider the time-out for the task split. By an appropriate choice of time-out to reduce the failure probability, no time-out assumption is a fair assumption.
} 
given below:

\section{Optimization Problem 1 (Optimal Split).}

$$
\begin{aligned}
& \boldsymbol{\phi}^{\star}=\underset{\phi}{\arg \min } \sum_{p=1}^{P}\left(\frac{a_{p} \phi_{p}^{2}}{r_{p}^{\text {comp }}-\phi_{p}}+\left(\frac{1}{r_{p}^{\text {comp }}}+\frac{1}{r_{p}^{\text {comm }}}\right) \phi_{p}\right), \\
& \text { s.t. } \quad \underline{\phi}_{p} \leq \phi_{p} \leq r_{p}^{\text {comp }} \quad \forall p \in\{1, \ldots, P\}, \text { and } \sum_{p=1}^{P} \phi_{p}=1,
\end{aligned}
$$

where the objective is minimizing the average job execution time. The constraints are: 1) $\phi$ must be a split (i.e. be positive and sum up to one); 2) all workers must be utilized at least to a certain amount $\underline{\phi}_{p}$; 3) each worker must have a stable queue.

Theorem 1 (Optimal Split). The solution of Optimization Problem 1 is given by,

$$
\phi_{p}^{*}= \begin{cases}\max \left\{r_{p}^{c o m p}\left(1-\sqrt{\frac{a_{p}}{a_{p}+\eta-\frac{1}{r_{p}^{c o m p}}-\frac{1}{r_{p}^{c o m m}}}}\right), \phi_{p}\right\}, & \frac{1}{r_{p}^{\text {comp }}}+\frac{1}{r_{p}^{\text {comm }}}-a_{p}<\eta, \\ \underline{\phi}_{p}, & \text { otherwise. }\end{cases}
$$

where $\eta$ is set such that $\sum_{p=1}^{P} \phi_{p}=1$.

Proof: The proof is given in Appendix B.

Theorem 1 is an analytical solution for Optimization Problem 1 . We note that $\phi_{p}$ is nondecreasing in terms of $\eta$, and thus one can do a binary search [35], [36] to identify $\eta$ with a desired precision such that $\sum_{p=1}^{P} \phi_{p}=1$.

Example 1. If workers are modeled with $M / M / 1$ queue, i.e., $a_{p}=1 / r_{p}^{\text {comp }}$, the optimal split is,

$$
\phi_{p}= \begin{cases}\max \left\{r_{p}^{\text {comp }}\left(1-\sqrt{\frac{\frac{1}{r_{p}^{\text {comp }}}}{\eta-\frac{1}{r_{p}^{\text {comm }}}}}\right), \underline{\phi}_{p}\right\}, & \frac{1}{r_{p}^{\text {comm }}}<\eta \\ \underline{\phi}_{p}, & \text { otherwise }\end{cases}
$$

where $\eta$ is set such that $\sum_{p=1}^{P} \phi_{p}=1$.

When the transmission delay is negligible, namely, $c_{p} \rightarrow \infty$ and consequently $r_{p}^{\text {comm }} \rightarrow \infty$, the optimal load split can obtained by the following according to Corollary 1, which is the solution for a special case of Optimization Problem 1 .

Corollary 1. When the communication delay is negligent, i.e., $c_{p} \rightarrow \infty$ and $r_{p}^{\text {comm }} \rightarrow \infty$, the 
optimal split is,

$$
\phi_{p}= \begin{cases}\max \left\{r_{p}^{c o m p}\left(1-\sqrt{\frac{a_{p}}{a_{p}+\eta-\frac{1}{r_{p}^{\text {comp }}}}}\right), \underline{\phi}_{p}\right\}, & \frac{1}{r_{p}^{\text {comp }}}-a_{p}<\eta, \\ \underline{\phi}_{p}, & \text { otherwise. }\end{cases}
$$

where $\eta$ is set such that $\sum_{p=1}^{P} \phi_{p}=1$.

Theorem 2 (Optimal Split for $r_{p}^{\text {comm }} \rightarrow \infty$ and exponential service time). Let assume the workers are sorted such that $r_{1}^{\text {comp }} \geq r_{2}^{\text {comp }} \geq \cdots \geq r_{P}^{\text {comp }}$. For $M / M / 1$ queuing model and when there is no communication delay, the optimal load split is obtained via,

$$
\phi_{p}= \begin{cases}r_{p}^{\text {comp }}\left(1-\sqrt{\frac{1}{\eta r_{p}^{c o m p}}}\right), & p \leq p^{*} \\ \underline{\phi}_{p} & \text { otherwise. }\end{cases}
$$

where $p^{*} \in\{1, \ldots, P\}$ is selected such that $\frac{r_{p^{*}}^{\text {comp }}}{\left(r_{p^{*}}^{\text {comp }}-\underline{\phi}_{p}\right)^{2}}<\eta\left(p^{*}\right) \leq \frac{r_{p^{*}+1}^{\text {comp }}}{\left(r_{p^{*}+1}^{\text {comp }}-\underline{\phi}_{p}\right)^{2}}, \eta=\eta\left(p^{*}\right)$, and the function $\eta\left(p^{+}\right)$is defined as follows,

$$
\eta\left(p^{+}\right) \triangleq\left(\frac{\sum_{p=1}^{p^{+}} \sqrt{r_{p}^{\text {comp }}}}{\sum_{p=1}^{p^{+}} r_{p}^{c o m p}+\sum_{p=p^{+}+1}^{P} \underline{\phi}_{p}-1}\right)^{2} .
$$

Proof: The proof is given in Appendix C

\section{B. Algorithm for Stream Distributed Coded Computing}

Given a set of code parameters, we first propose in our solution how to select a set of workers that provide sufficient computational resources for the setting, i.e., they are valid and their cumulative task service rate holds Assumption 2 with a margin $\Theta$ for the $(\Omega-1) K$ supportive tasks per job (See Definition 1).

Optimization Problem 2. Let $\overline{\mathcal{P}}$ be a large set of available workers that are sorted in a decreasing order according to their computational power. The subsets of workers $\mathcal{P} \subset \overline{\mathcal{P}}$ that are acquired for the distributed computation are the first valid $P$ workers in $\overline{\mathcal{P}}$ such that,

$$
\sum_{p=1}^{P} r_{p}^{\text {comp }} \geq 1+\Theta \quad \text { s.t. } \quad \Theta \geq(\Omega-1) .
$$

We remind that $r_{p}^{\text {comp }}=1 / \lambda E\left[T_{p}\right]$. We assume the set of given workers provide sufficient combined computational power for the job arrival rate $\lambda$, and the above optimization problem is feasible. We remind that $E\left[T_{p}\right], p \in\{1, \ldots, P\}$, depends on the code parameters. Denoting by $\mathcal{C}$ the set of options for the code parameters, each element of $\mathcal{C}$ corresponds to a choice for parameters in $\left\{K, I_{\text {in }}, I_{\text {out }}, C, E\left[T_{\text {enc }}\right], E\left[T_{\text {dec }}\right]\right\}$. 
The overall solution can be described as follows: For any member of the set $\mathcal{C}$ (the choices for the code parameters), a subset of workers is selected according to Optimization Problem 2 Then, the optimal load split $\left\{\phi_{1}, \ldots, \phi_{P}\right\}$ are identified using Optimization problem 1 , and the corresponding average job execution time $D_{\text {exe }}$ is recorded. The best valid member of the set $\mathcal{C}$ (resulting in the minimum job execution) along with the corresponding optimal load split are recorded for utilization. The procedure is shown in Algorithm 1, and run in initial stage at the master node or when changes occur in the system, e.g., according to the feedback, due to a worker unavailability, etc.

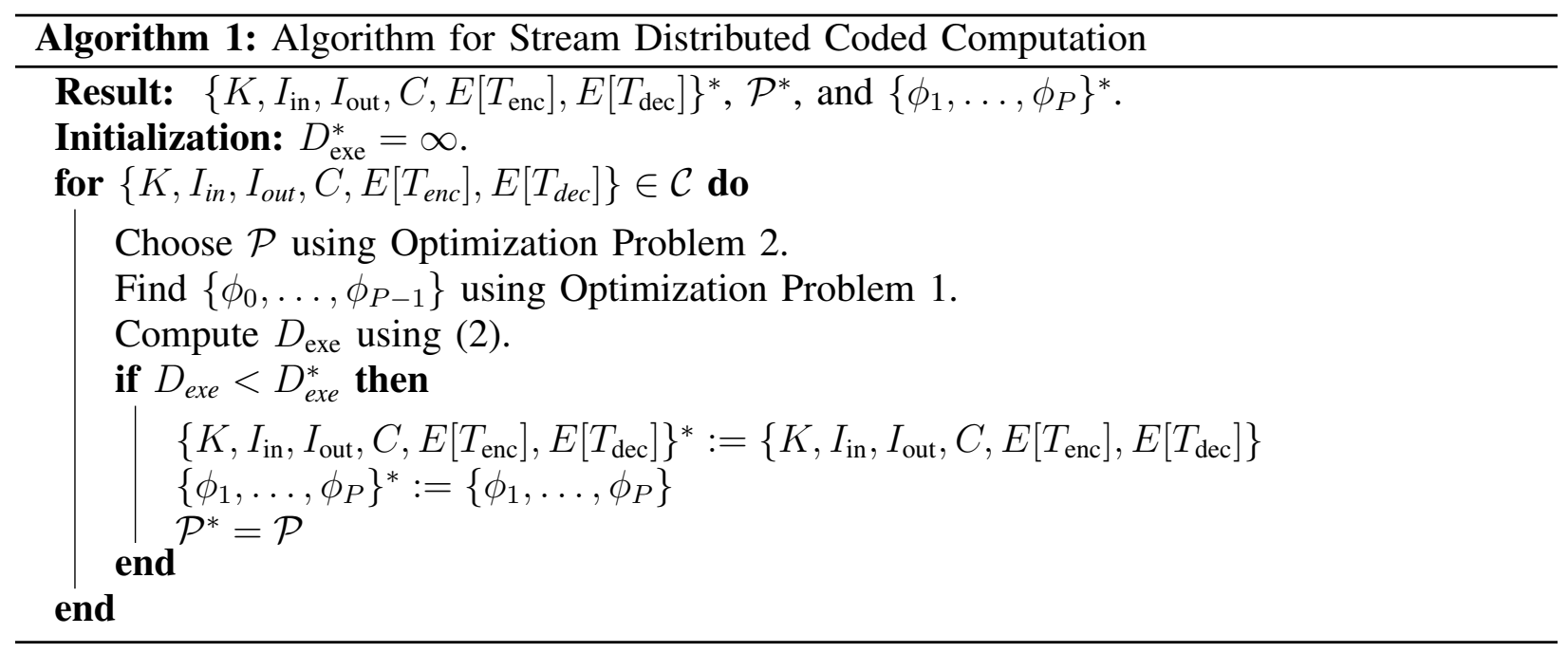

Example 2 (Matrix Multiplication using PolyDot). For the PolyDot codes scheme, with any positive integers $s$ and to split the matrices as defined in Appendix A the set of codes can be identified as follows:

$$
\mathcal{C}=\{(s, t) \mid 1 \leq s, t \leq m \text { and } s t=m\}
$$

The other code parameters are given in terms of $s$ and $t$, as follows:

- The number of critical tasks is $K=t^{2}(2 s-1)$.

- The master node transmits $I_{\text {in }}=2 K \Omega N^{2} /$ st symbols to designated workers per job, and the workers transmit $I_{\text {out }}=K \Omega N^{2} / t^{2}$ symbols to the fusion node $]^{6}$

- The computational complexity of each job is $C=K \Omega N^{3} / s t^{2}$.

- The average encoding time is $E\left[T_{\text {enc }}\right]=K \Omega N^{2} / \mu_{\text {enc }}$, and the average decoding time is $E\left[T_{d e c}\right]=\left(N^{2} K+K^{3}\right) / \mu_{\text {dec }}$. Here $\mu_{\text {enc }}$ and $\mu_{\text {dec }}$ denote the computational power of the master node and fusion node to perform computational powers 7

\footnotetext{
${ }^{6}$ In case of purging, $I_{\text {out }}=K N^{2} / t^{2}$.

${ }^{7}$ Note that these computational complexities correspond to specific encoding and decoding algorithms. Information regarding other encoding and decoding algorithms can be found in [26].
} 


\section{FeEdBack-BASEd Tracking}

In this section, we propose two feedback-based algorithms to track the state of workers and adjust the system parameters accordingly. The first algorithm given in Section $\mathrm{V}-\mathrm{A}$ is for adaptive estimation of workers' statistical features. The second algorithm given in Section $\mathrm{V}-\mathrm{B}$ is for posteriori job reinforcement at the workers.

\section{A. Adaptive Estimation of Workers' Computational Statistical Features}

One key feature of our solution is the adaptive feature of the load assignments based on the time-varying average service rate of the workers. To this end, the master node requires to have access to the system parameters $E\left[T_{p}\right]$ and $E\left[T_{p}^{2}\right], p \in\{1, \ldots, P\}$. These parameters are provided by the workers' declaration but are adjusted during the execution according to system realizations. In this subsection, we propose several efficient estimators for this purpose, and the right choice depends on the desired trade-offs among accuracy, information availability, and computational limits. Let $U_{p}$ be the task service time of the $p$-th worker, $p \in\{1, \ldots, P\}$, which is the time it takes for the $p$-th worker to finish a task. The master node, through feedback, has access to realizations of $U_{p}$ over the time, and intends to adjust the estimations of $E\left[T_{p}\right]$ and $E\left[T_{p}^{2}\right]$ accordingly.

Let parameters $E_{p}(l)$ and $S_{p}(l)$ be estimations of first and second moments of $U_{p}$ at time $l$. We identify $E_{p}(l)$ and $S_{p}(l)$ according to realizations of $U_{p}$ up to and including time $l$, i.e., $\left\{u_{p}(1), \ldots, u_{P}(l)\right\}$,

$$
\begin{aligned}
E_{p}(t) & :=(1-\alpha) E_{p}(l-1)+\alpha u_{p}(l), \\
S_{p}(t) & :=(1-\beta) S_{p}(l-1)+\beta u_{p}^{2}(l) .
\end{aligned}
$$

These estimation rules are based on the considered problem setting where the first moment and second moment of the time it takes for the $p$-th worker to finish $\phi_{p}$ portion of a job is $\phi_{p} E\left[T_{p}\right]$ and $\phi_{p}^{2} E\left[T_{p}^{2}\right]$, respectively, and one can consider a different appropriate estimation rules for a different problem setting. Here, $\alpha$ and $\beta$ are forgetting factors that determine the importance of old realizations in estimations. If $\alpha=\beta=1 / l$, then all previous samples have equivalent importance in estimations.

Alternatively, the parameters $E_{p}(l)$ and $S_{p}(l)$ can be updated each $\Lambda$ time steps according to the following rules,

$$
\begin{aligned}
& E_{p}(l):=(1-\alpha)^{\Lambda} E_{p}(l-\Lambda)+\sum_{i=1}^{\Lambda}(1-\alpha)^{\Lambda-i} \alpha^{i} u_{p}(l-i+1), \\
& S_{p}(t):=(1-\beta)^{\Lambda} S_{p}(l-\Lambda)+\sum_{i=1}^{\Lambda}(1-\beta)^{\Lambda-i} \beta^{i} u_{p}^{2}(l-i+1) .
\end{aligned}
$$


Finally, considering each job consists of $K \Omega$ tasks with no purging, we adjust estimations of $E\left[T_{p}\right]$ and $E\left[T_{p}^{2}\right]$ at time step $l$ as follows,

$$
E\left[T_{p}\right]=\frac{E_{P}(l)}{K \Omega}, \quad \text { and } \quad E\left[T_{p}^{2}\right] \simeq \frac{S_{P}(l)}{K^{2} \Omega^{2}} .
$$

The approximation comes from the fact that, in practice, the tasks are performed one by one at the workers. Thus, the time it takes for the $p$-th worker to perform one job is the time it takes for the $p$-th worker to perform $\phi_{p} K \Omega$ sequential tasks which is summation of several random variables that may have different distribution than the distribution of one of these random variables. According to our empirical results and analytical analysis, these approximations are realistic especially when $K \Omega \underline{\phi}_{p} \gg 1, p \in\{1, \ldots, P\}$.

Example 3. Consider $U_{p}, p \in\{1, \ldots, P\}$, which is the time it takes for the $p$-th worker to finish a task, has an exponential distribution with parameter $K \Omega \tilde{\mu}_{p} / C$. Then, $T_{p}\left(\phi_{p}\right), p \in\{1, \ldots, P\}$, which is the time it takes for the $p$-th worker to finish $\phi_{p}$ portion of a job or equivalently $K \Omega \phi_{p}$ tasks, i.e., has a Gamma distribution with shape $K \Omega \phi_{p}$ and scale $C / K \Omega \tilde{\mu}_{p}$. In this setting, we have

$$
E\left[T_{p}\left(\phi_{p}\right)\right]=\phi_{p} \frac{C}{\tilde{\mu}_{p}}=\phi_{p} E\left[T_{p}\right], \quad \text { and } \quad E\left[T_{p}\left(\phi_{p}\right)^{2}\right]=\phi_{p}\left(\phi_{p}+\frac{1}{K \Omega}\right) \frac{C^{2}}{\tilde{\mu}_{p}^{2}} \simeq \phi_{p}^{2} E\left[T_{p}^{2}\right],
$$

The above approximation is valid if $\phi_{p} \gg 1 / K \Omega, p \in\{1, \ldots, P\}$. Since, we have $\phi_{p} \geq \underline{\phi}_{p}$, then $K \Omega \underline{\phi}_{p} \gg 1$ ensures the approximations are valid.

Moreover, the considered $T_{p}$ as above estimation is an upper bound since the fusion node only requires $K$ task results to obtain the final job result. In case, the master node remove the tasks related to a resolved job from the queue of the master node (purging), each job consists of less then $K \Omega$ tasks. In this case, one can incorporate the purging probability into the estimation and refine the analysis.

\section{B. Feedback-Based Algorithm for Posteriori Job Reinforcement}

In the distributed computation scheme considered, the master node tracks the service rate of workers via the feedback from the workers or fusion node. The optimal task split optimization enables us to optimally split the $K \Omega$ tasks amongst the workers at the time the job arrives. Here, we propose an efficient posteriori adaptive algorithm to adjust the load split according to the workers' current service rate during the job's computation period. The adaptive algorithm proposed can minimize the in-order delivery delay of jobs while maximizing the utilization of the workers.

Let $\mathcal{A}_{j}(l)$ be a counter at the master that records the number of successful task results related to the $j$-th job up to and including time step $l$, obtained using the feedback information. We consider the case that $\mathcal{A}_{j}(l)<K$, and thus the job is not resolved at the fusion node. We denote 
as $l_{j}$ the time that $j$-th job arrives at the master node and is distributed among the workers, and $l>l_{j}$ the time that a feedback related to $j$-th job is received by the master node. Besides, $E_{l}\left[T_{p}\right]$ is the estimation of the job service time for the $p$-th worker at time step $l$. We also denote with $\mathcal{P}(l, j)$ the set of workers that are working on $j$-th job at time step 8 . We define $\mu_{E}^{j}(l)$ and $\mu_{R}^{j}(l)$ as the expected computation rate and realized computation rate of $j$-th job for the workers in $\mathcal{P}(l, j)$, and they are obtained as follows

$$
\mu_{E}^{j}(l) \triangleq \frac{1}{|\mathcal{P}(l, j)|} \sum_{p \in \mathcal{P}(l, j)} \frac{\phi_{p}}{E_{l_{j}}\left[T_{p}\right]}, \quad \mu_{R}^{j}(l) \triangleq \frac{1}{|\mathcal{P}(l, j)|} \sum_{p \in \mathcal{P}(l, j)} \frac{\phi_{p}}{E_{l}\left[T_{p}\right]} .
$$

Here $\left\{\phi_{1}, \ldots, \phi_{p}\right\}$ are based on the optimal task split upon arrival of the $j$-th job.

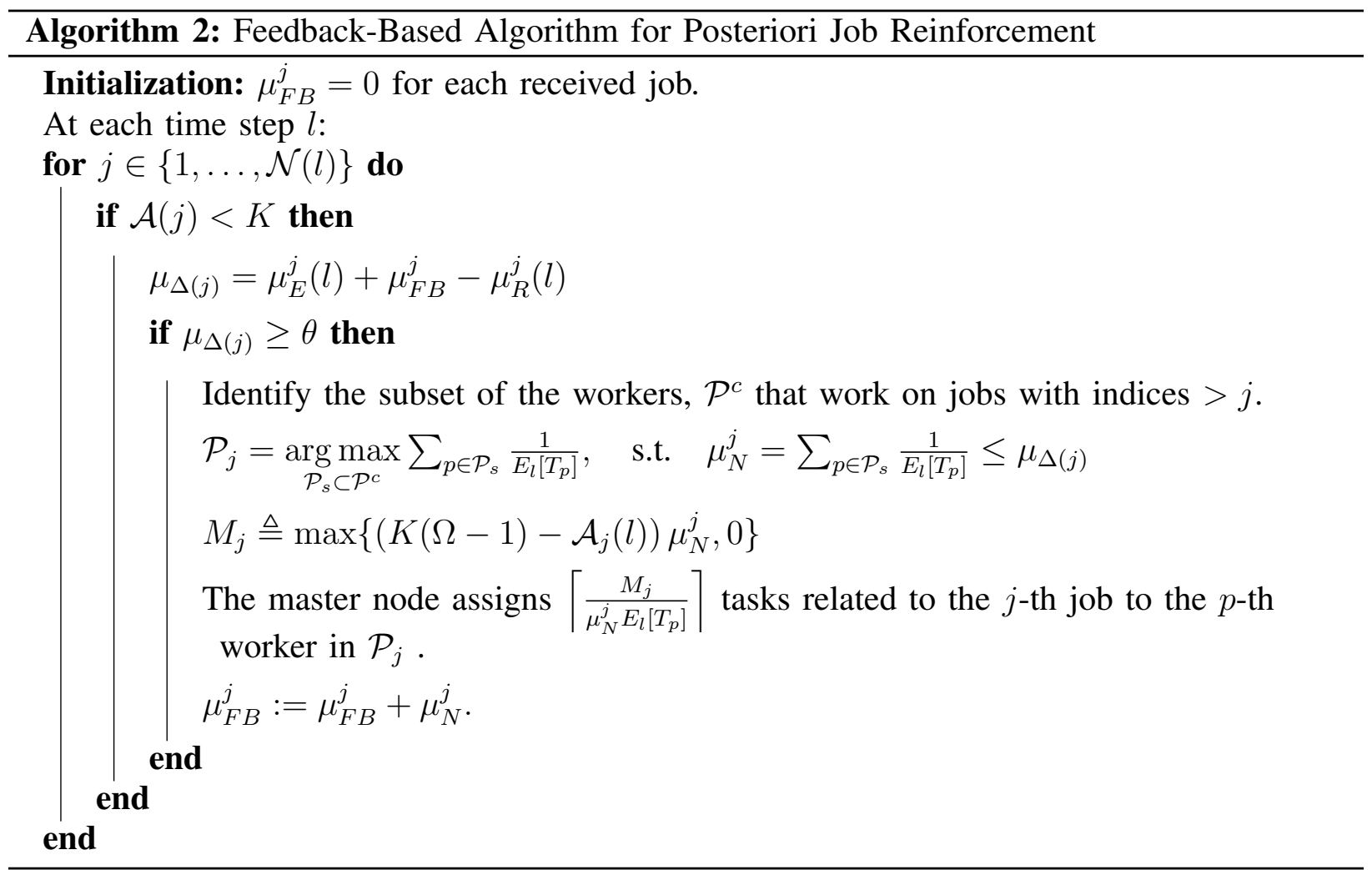

Then, $\mu_{R}^{j}(l)-\mu_{E}^{j}\left(l_{j}\right)$ quantifies the missing service rate due to the stragglers related to the $j$-th job up to and including time step $l$. Upon arrival of any feedback related to the $j$-th job at time step $l, \mu_{\Delta(j)}$ is computed according to the following rule,

$$
\mu_{\Delta(j)}=\mu_{E}^{j}(l)+\mu_{F B}^{j}-\mu_{R}^{j}(l),
$$

${ }^{8}$ If $l>l_{j+1}$, these set of workers are the stragglers for the $j$-th job 
where the parameter $\mu_{F B}^{j}$ indicates the adjusted computation rate that have been considered so far for the $j$-th job according to feedback. Then, if $\mu_{\Delta(j)}>\theta$, (here $\theta$ is a pre-defined threshold), the master node attempts to assign appropriate number of reinforcing tasks related to this job to an appropriately selected subset of workers $\mathcal{P}_{j}$ at beginning of their queue.

Let $\mathcal{P}^{c}$ be a subset of workers that have started to work on subsequent jobs 9 , i.e.,

$$
\mathcal{P}_{j}^{c}=\mathcal{P} \backslash \mathcal{P}(l, j) \backslash \mathcal{P}(l, j-1) \backslash \cdots \backslash \mathcal{P}(l, 1) .
$$

The appropriately selected subset of workers $\mathcal{P}_{j}$ to reinforce the $j$-th job is obtained using the following optimization problem,

$$
\mathcal{P}_{j}=\underset{\mathcal{P}_{s} \subset \mathcal{P}^{c}}{\arg \max } \sum_{p \in \mathcal{P}_{s}} \frac{1}{E_{l}\left[T_{p}\right]}, \quad \text { s.t. } \quad \mu_{N}^{j}=\sum_{p \in \mathcal{P}_{s}} \frac{1}{E_{l}\left[T_{p}\right]} \leq \mu_{\Delta(j)}
$$

Then, the parameter $M_{j}$ is set as follows,

$$
M_{j} \triangleq \max \left\{\left(K(\Omega-1)-\mathcal{A}_{j}(l)\right) \mu_{N}^{j}, 0\right\} .
$$

which shows the number of reinforcing tasks for the $j$-th job. Next, $\mu_{F B}^{j}$ is updated as follows,

$$
\mu_{F B}^{j}:=\mu_{F B}^{j}+\mu_{N}^{j} .
$$

The adaptive posteriori procedure is given as Algorithm 2. Note that in solutions considered in the literature (e.g. in [3], [4], [26]) and also here in previous sections without the adaptive algorithm, if some workers stop/halt during the execution, i.e., their service rate approaches zero for some period of time, the system will be prone to some failure probability. Using the proposed adaptive algorithm, we will compensate for this missing service rate due to the workers that stop to work by other workers.

\section{NumericAl RESUlTS AND Discussion}

In this section, we evaluate the performance of the proposed joint coding and scheduling framework for stream distributed coded computation. We first evaluate the method for selecting a subset of the workers from the available ones $\overline{\mathcal{P}}$. Then, we compare the performance of our framework in light of the trade-off between computational load and execution time. The coding scheme we consider here is the PolyDot code (see Appendix A and Example 2) with parameters $N=100$, $m=50$. Therefore, the set of all codes can be described as $\mathcal{C}=\{(s, m / s) \mid m$ is divisible by $s\}$. For sake of illustration's clarity, we have considered $s$ can be any integer in $\{1, \ldots, m\}$, and in practice, one should pick a value of $s$ that is closest to the optimal value and divides $m$. In this set of simulations, the task service time of each worker is drawn for an exponential distribution

\footnotetext{
${ }^{9}$ It can also include the workers that have worked faster than expected, i.e., $E\left[T_{p}\right]<E_{j}\left[T_{p}\right]$.
} 

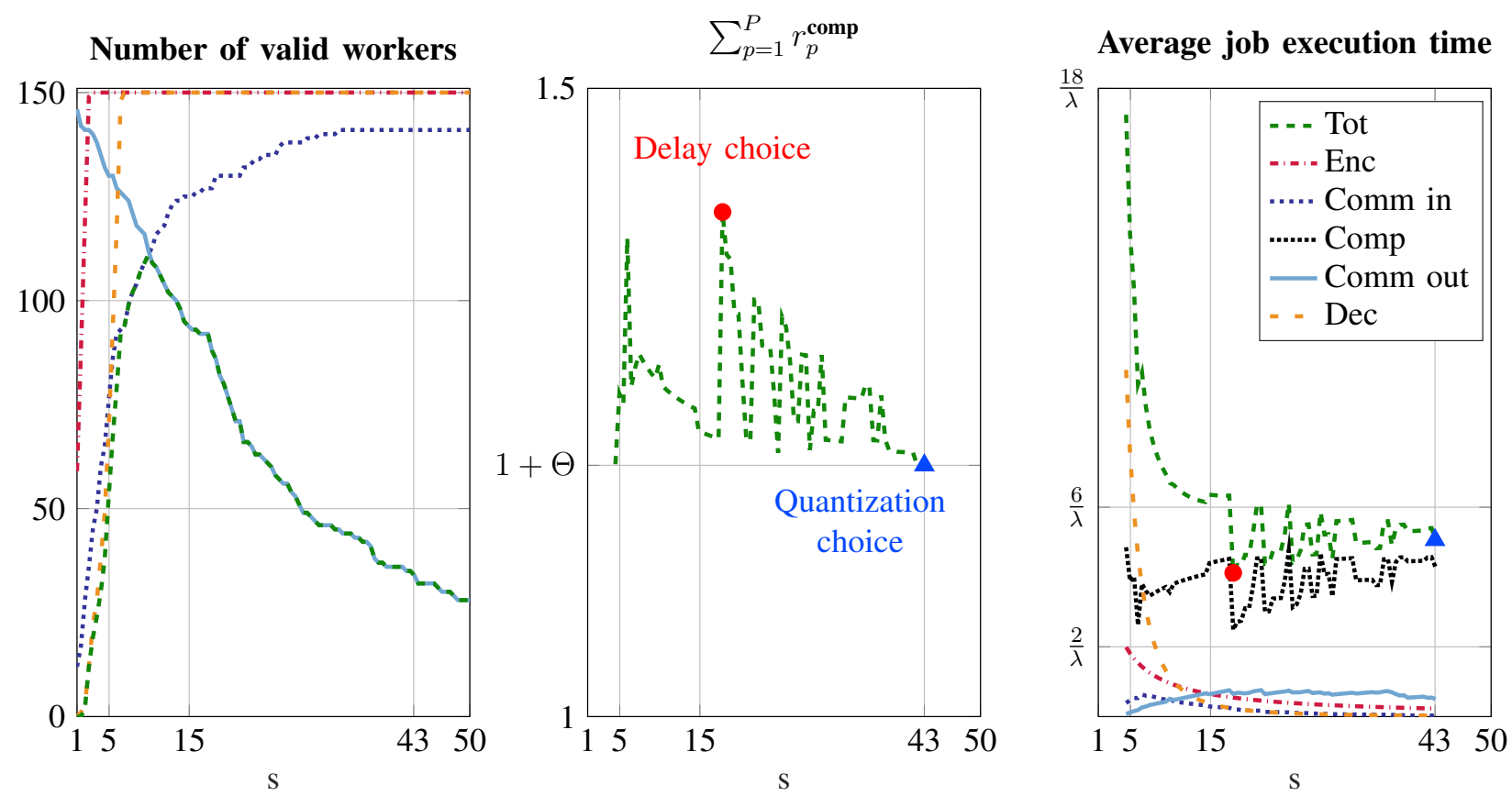

Fig. 3: Exploring the effect of the initial set of workers on the system parameters and performance. (a): The number of valid and partially valid workers. (b): Total computational rate of the selected workers versus the code parameter $s$. (c): The average job execution time versus the code parameter $s$, along with its constituent parts, when the stability conditions hold.

with rate parameter $\tilde{\mu}_{p}$ operations per time step. As discussed in Example 3, this results in a job service time following a Gamma distribution.

\section{A. Evaluation of the Initial Choice of workers}

First, we illustrate and evaluate the impact of our method for choosing the workers. In this set of simulations, the rate parameter $\tilde{\mu}_{p}$ of each worker is uniformly and independently selected in $[0,1000]$ operations per time step, the transmission rate $c_{p}$ of each worker is uniformly and independently selected in [0,200] symbols per time step, $\mu_{\mathrm{enc}}=10000$ operations per time step, and $\mu_{\mathrm{dec}}=100000$ operations per time step. Given the system parameters, one can easily find $1 / E\left[T_{p}\right], c_{p} / I_{\text {in }}$, and $c_{p} / I_{\text {out }}$ for each worker in $\overline{\mathcal{P}}$, along with $1 / E\left[T_{\text {enc }}\right]$ and $1 / E\left[T_{\text {dec }}\right]$. We remind that the set of valid workers is a subset of $\overline{\mathcal{P}}$ such that computation is the bottleneck for each one of them. In other words, the $p$-th worker in $\overline{\mathcal{P}}$ is valid if $1 / E\left[T_{p}\right]$ is smaller than $c_{p} / I_{\text {in }}$, $c_{p} / I_{\text {out }}, 1 / E\left[T_{\text {enc }}\right]$, and $1 / E\left[T_{\mathrm{dec}}\right]$, see Definition 2 .

Fig. 3(a) explores a realization of $\overline{\mathcal{P}}$ that consists of 150 workers with respect to the code parameter $s$. The red dashed-dotted curve shows the number of workers such that their computation rate is lower than the encoding rate, i.e., $\left\{p \in \overline{\mathcal{P}} \mid 1 / E\left[T_{p}\right]<1 / E\left[T_{\text {enc }}\right]\right\}$. Similarly, the dashed dark blue, resp. solid light blue and dashed orange, curve shows the number of workers such that their computation rate is lower than their incoming traffic rate, resp., outgoing traffic 
rate and the decoding rate. The dashed green curve shows the number of valid workers which stands below all these curves. We observe that for small values of $s$, the decoding defines an upper bound for the number of valid workers, while for large values of $s$, the outgoing traffic rate defines an upper bound. The highest number of valid workers is obtained when $s=10$.

Once the valid workers are identified, a subset of them is selected to serve the queue of in-order jobs at the master node, using Optimization Problem 2. Fig. 3(b) shows the value of $\sum_{p=1}^{P} r_{p}^{\text {comp }}$, which must be equal or greater than $1+\Theta$, with respect to the code parameter $s$ when $\lambda=10^{-3}$. We exclude those values of $s$ such that the valid workers do not provide sufficient resources to serve the queue of the jobs, i.e. when Optimization Problem 2 is not feasible. This happens in this example when $s<5$ or $s>43$. Thus, we restrict ourselves to admissible values of $s$ that result in feasible optimizations.

The optimal choice of $s$ is the one that results in the minimum average job execution time. Fig. 3 (c) shows the average job execution time with dashed green line for various admissible values of $s$. Moreover, it shows the constituent components of the average job execution time, i.e., encoding time, communication time from the master node to workers, computation time at the workers, communication time from the workers to the fusion node, and decoding time. We observe that the average job execution time with respect to $s$ has several local minima, and selecting any of them can be a viable choice. The fluctuations in the curve is due to the quantization errors that enter into the design in Optimization Problem 2 . Hence, in this example, choosing any value of $s$ between 15 and 43 is reasonable. Several criteria can be considered for selecting the optimal $s$. First, one can choose $s$ leading to the minimum delay (computation time), as depicted by a red point in the figure. This case will likely correspond to the highest quantization error (i.e., reduced utilization). Another choice is to select $s$ such that the delay remains reasonable while minimizing the quantization error (i.e., maximum utilization), as depicted by a blue triangle in the figure. Finally, one should also take into account that $s$ must divide $m$ in order to obtain a valid code.

\section{B. Trade-off between computational load and average job execution time}

Here, we investigate the performance of our framework with respect to the performances of two extreme baselines: 1) Naive load split where the computational load of a job is distributed uniformly among several workers regardless of their differences. 2) Ideal load split 10 where the master node gets informed when a worker finishes its assigned task and outsources another task to it. In this set of simulations, the rate parameter $\tilde{\mu}_{p}$ of each worker is uniformly and independently selected in $[0,2500]$ operations per time step, the transmission rate $c_{p}$ of each

\footnotetext{
${ }^{10}$ Since, in our work, we assume the communication delay exists, the ideal split works in a non-causal way such that the next task arrives precisely when the worker becomes idle.
} 


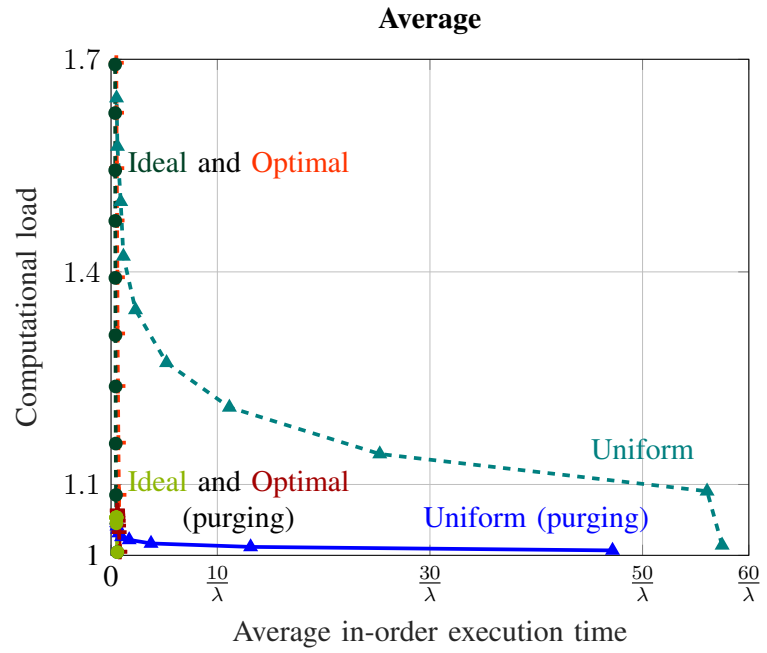

(a) Average execution time versus computational load

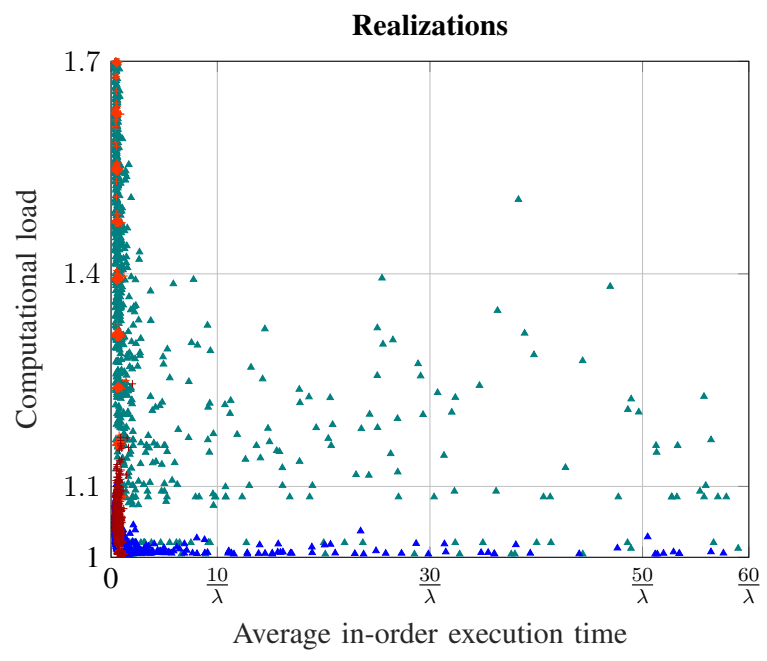

(c) Realizations of execution time versus computational load

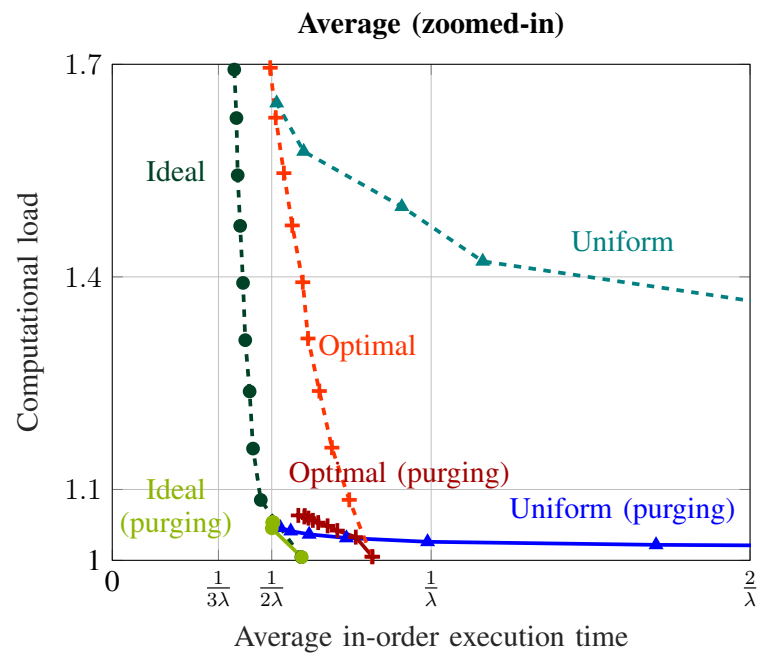

(b) Average execution time versus computational load Realizations (zoomed-in)

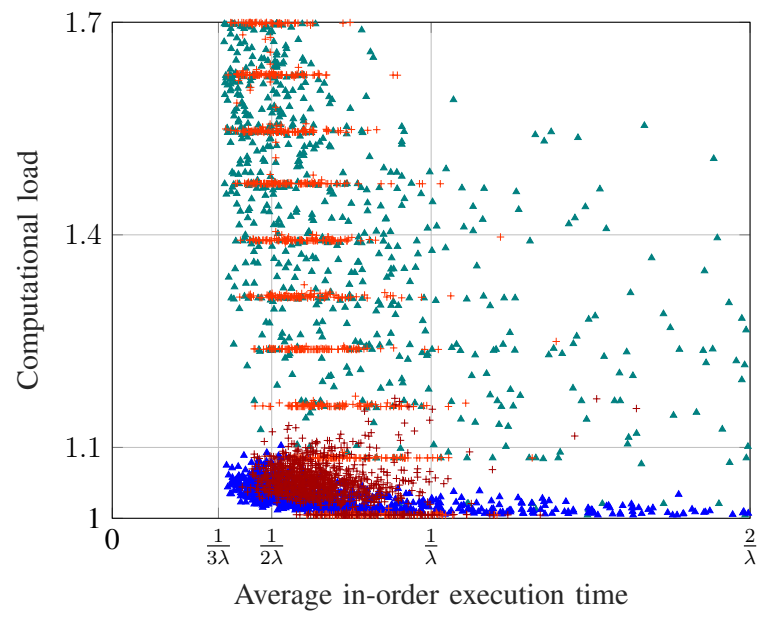

(d) Realizations of execution time versus computational load

Fig. 4: Trade-off between computational load and job execution time for a cluster of heterogeneous workers. The right figures are zoomed-in versions of the left ones. The bottom figures highlight 100 realizations out of the 500 which have been used to obtain the average execution time for the above ones (For the sake of clarity, the realizations of the ideal case have not been drawn.). The performances have been averaged over 500 realizations with 200 jobs each, $\lambda=10^{-3}, m=50, N=100, s=40, t=10, \Theta=2$, and the forgetting factor of the mean and variance estimations equal to 0.01 . The parameter $\Omega$ changes in $[1,1.7]$. Finally, we note that $\frac{1}{3 \lambda}$ is the inverse of the total service rate. This delay corresponds to the computation delay (i.e. neglecting communication, encoding and decoding) when jobs are served instantaneously (i.e. neglecting the time in the queue). 
worker, is uniformly and independently selected in $[0,1000]$ symbols per time step, $\mu_{\mathrm{enc}}=10^{5}$ operations per time step, and $\mu_{\mathrm{dec}}=10^{6}$ operations per time step ${ }^{11}$.

We select $\Theta=2$, i.e., $\sum_{p=1}^{P} r_{p}^{\text {comp }}=3$, and consequently, the set of workers can serve a queue of jobs with average arrival rate up to $3 \lambda$. In fact, $\Theta$ affects the number of feasible solutions for Optimization Problem 1, and a lower $\Theta$ lowers the impact of the optimal load split. We first remind that $D_{\text {exe }}$ is the average execution time per job, and $\Omega$ determines the computational load, i.e., the ratio of the total amount of computations (including the redundant computations) to necessary amount of computations. Fig. 4 depicts the computational load versus average job execution time for various interesting scenarios where $\lambda=10^{-3}$ and the redundancy ratio $\Omega$ varies in interval $[1,1.7]$. We highlight that $\sum_{p=1}^{P} r_{p}^{\text {comp }}$, i.e., the total computational rate of the workers per job, depends on $\Omega$, which determines the number of tasks per job and the selected set of workers to serve $K \Omega$ tasks by job.

We first note that the uniform split, i.e., $\phi_{p}=1 / P$ and $p \in\{1, \ldots, P\}$, does not necessarily result in a stable solution as confirmed by our simulation results. The ideal split, i.e., genieaided, has a time variant load split, such that the master node assigns a task to a worker as soon as the worker declares the previous task has finished. Lastly, the optimal split, is obtained using the proposed framework in this paper. Purging is an optimal feature where the master node asks all workers to drop their tasks related to a job once the decoder is able to decode the job result using the successful tasks up to that time. Fig. 4 shows the trade-off between the average job execution time and the computational load for the various split schemes with and without purging. In case, there is no purging, the computational load scales proportional to $\Omega$ as all tasks are computed ${ }^{12}$. When there is purging, the computational load gets closer to 1.

As we see in Fig. 4, the average execution time of the uniform split is much larger than the optimal and ideal splits. This is because a subset of workers are unstable in this naive split. When the computational load increases, the average job execution time for the uniform scheme decreases since the supportive task results of the stable workers can compensate for the unstable workers. More importantly, we observe that the performance of the proposed optimal split is close to the performance of the genie-aided split, although our approach relies on negligible feedback from the fusion node for estimations. From Fig. 4(c) and (d), which show the realizations of 100 of the 500 independent runs which have been used to generate Fig. 4(a) and (b), one can also observe the reliability of our approach with respect to the uniform split method. Indeed, not only the average performance is better with our framework, but also all runs have close performances. This is very different from the uniform case where the choice of the workers greatly impact the performance.

\footnotetext{
${ }^{11}$ In this set of simulations, we do not use Optimization Problem 2 to select the workers to investigate a heterogeneous cluster of workers. Hence, we are facing fast and slow workers.

${ }^{12}$ The uniform solution seems to have a lower computational load than the other methods at high $\Omega$. This is a simulation artefact: as the queues are not stable, the simulation is ended before all unnecessary tasks of the unstable workers are computed. Hence, this translates into a utilization which does not scale exactly as $\Omega$.
} 


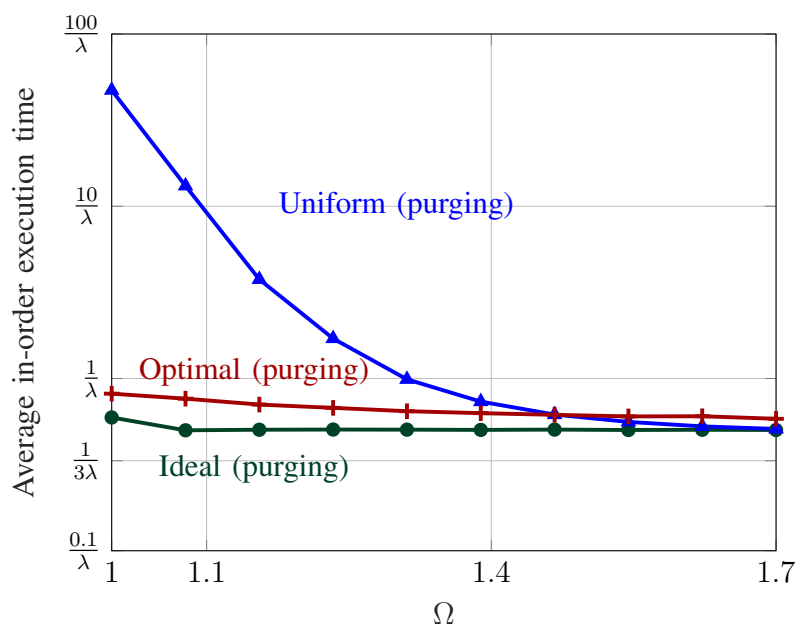

Fig. 5: Evolution of the in-order execution time with respect to the redundancy ratio $\Omega$. These results are exactly those of Fig. 4, yet presented in a different way.

In all cases, when purging is performed, the computational load decreases and approaches to one. We see that when $\Omega=1$, purging has no effect since all tasks are necessary to resolve a job. However, the computational load slightly increases when $\Omega$ increases as more workers are used, and therefore more tasks are likely to arrive at the fusion node before purging is performed. In case of purging and when $\Omega$ is large, the performance of genie-aided, uniform, and optimal split get closer. Interestingly, our optimal solution has a slightly bigger average execution time than the two others for high $\Omega$, with purging. This can be explained by the fact that the optimization problem, based on a queuing model, does not incorporate the purging mechanism into its delay analysis. To reduce this degradation, one can incorporate purging probability into the estimation and refine the analysis.

As the computational load remains close to one, in order to better grasp the impact of $\Omega$ on the execution time, Fig. 5 shows the evolution of the average in-order execution time as a function of $\Omega$. We again see that our optimal solution remains very close to the ideal non-causal one for all values of $\Omega$. As discussed above, the discrepancy between the three different solutions vanishes at high $\Omega$ since tasks are purged before entering into the regime where the optimization is useful.

\section{CONCLUSIONS}

In this paper, we studied a stochastic heterogeneous setting for the stream distributed coded computation problem. We proposed a systematic framework for the joint scheduling-coding that incorporates the diverse properties of the workers into the design. In particular, an appropriate set of workers from a pool of workers are chosen to provide stability, and the coding parameters and the load split among the selected workers are optimally identified to minimize the average 
in-order job execution time. Furthermore, a realistic feedback model was introduced to track the state of the workers and the progress of ongoing jobs at the master node.

An interesting future direction is to extend the framework for stream computation of jobs with general arrival model, not necessarily Poisson arrival model. Moreover, proposing a joint scheduling-coding for stream distributed computation in a network of heterogeneous interconnected workers is another interesting future research direction. Last but not the least, generalizing the proposed framework for stream computation of iterative jobs, often required in machine learning applications, is one of our future goals.

\section{ACKNOWLEDGMENTS}

This work is supported by the European Regional Development Fund (FEDER), through the Regional Operational Programme of Centre (CENTRO 2020) of the Portugal 2020 framework and FCT under the MIT Portugal Program [Project SNOB-5G with Nr. 045929(CENTRO-010247-FEDER-045929)]. Guillaume Thiran is a Research Fellow of the F.R.S.-FNRS.

\section{APPENDIX A}

\section{Distributed Matrix Multiplication with Polydot}

Here, we provide background on distributed matrix multiplication with PolyDot scheme [26]. The computational job is multiplication of two large matrices $\mathbf{A}$ and $\mathbf{B}$ of size $N \times N$, i.e., $\mathbf{A B}$, in a distributed fashion. The computational inputs of each job are devised as follows:

$$
\mathbf{A}=\left[\begin{array}{ccc}
\mathbf{A}_{0,0} & \cdots & \mathbf{A}_{0, s-1} \\
\vdots & \ddots & \vdots \\
\mathbf{A}_{t-1,0} & \cdots & \mathbf{A}_{t-1, s-1}
\end{array}\right], \quad \mathbf{B}=\left[\begin{array}{ccc}
\mathbf{B}_{0,0} & \cdots & \mathbf{B}_{0, t-1} \\
\vdots & \ddots & \vdots \\
\mathbf{B}_{s-1,0} & \cdots & \mathbf{B}_{s-1, t-1}
\end{array}\right]
$$

$s t=m, X_{s i+j}=\left[\mathbf{A}_{i, j}, \mathbf{B}_{i, j}\right]$, and $f\left(X_{1}, \ldots, X_{m}\right)=\mathbf{A B}$. The master node splits each multiplication job into a set of smaller tasks. The inputs of $r$-th task has the format $\left[\mathcal{P}_{\mathbf{A}}(x), \mathcal{P}_{\mathbf{B}}(x)\right]$, evaluated at $x=x_{r}$, and will be sent to a worker to perform the multiplication over these smaller matrices. The matrices $\mathcal{P}_{\mathbf{A}}(x)$ and $\mathcal{P}_{\mathbf{B}}(x)$ are defined as follows:

$$
\mathcal{P}_{\mathbf{A}}(x)=\sum_{i=0}^{t-1} \sum_{j=0}^{s-1} \mathbf{A}_{i, j} x^{t j+i}, \quad \mathcal{P}_{\mathbf{B}}(x)=\sum_{k=0}^{s-1} \sum_{l=0}^{t-1} \mathbf{B}_{k, l} x^{t(2 s-1) l+t(s-1-k)} .
$$

Then, the worker computes $\mathcal{P}_{\mathbf{C}}\left(x_{r}\right)=\mathcal{P}_{\mathbf{A}}\left(x_{r}\right) \mathcal{P}_{\mathbf{B}}\left(x_{r}\right)$. Fusion node uses $K=t^{2}(2 s-1)$ successful task results from the workers to identify the coefficient of term $x^{t(2 s-1)(l-1)+t(s-1)+i-1}$ for polynomial $\mathcal{P}_{\mathbf{C}}(x), \forall i, l \in\{0, \ldots, t-1\}$, defined below:

$$
\mathcal{P}_{\mathbf{C}}(x)=\sum_{i, j, k, l} \mathbf{A}_{i, j} \mathbf{B}_{k, l} x^{t(2 s-1) l+t(s-1+j-k)+i} .
$$


Note this coefficient, which happens when $j-k=0$, is $\mathbf{C}_{i, l}=\sum_{k=0}^{s-1} \mathbf{A}_{i, k} \mathbf{B}_{k, l}$. The maximum degree of $p_{\mathbf{C}}(x)$ is $t^{2}(2 s-1)-1$, thus one needs $t^{2}(2 s-1)$ evaluations to identify the polynomial.

The parameters $K, I_{\text {in }}, I_{\text {out }}, C$, and the encoding and decoding computational complexities are functions of parameter $s$ and $t$, as we see in Example 2. These parameters influence the average job execution time, and we later optimize these two parameters to achieve a desired trade-off between various performance metrics of our solution.

\section{APPENDiX B}

\section{Proof of TheOREM 1 - Optimal SPLit 1}

The proof of Theorem 1 is considered here. We first show that the Optimization Problem 1 is convex. Hence, the optimal solution is unique.

Lemma 1. The objective function in (3) is strictly convex on its domain, i.e.,

$$
\phi \in\left\{\left\{\phi_{1}, \ldots, \phi_{P}\right\} \mid 0 \leq \phi_{p} \leq r_{p}^{c o m p}\right\}
$$

Proof. The objective function in (3) is summation of $P$ strictly convex terms and $P$ linear terms, and thus it is strictly convex. We note $f(\phi)=\frac{\alpha \phi^{2}}{\beta-\phi}$ is strictly convex over $\phi_{p} \leq \phi \leq \beta$ when $\alpha>0$ since,

$$
f^{\prime \prime}(\phi)=\frac{2 \alpha}{\beta-\phi}+\frac{2 \alpha \phi(2 \beta-\phi)}{(\beta-\phi)^{3}}>0, \quad \forall 0 \leq \phi \leq \beta
$$

Since the optimization problem has a strictly convex objective function, and the Slater's conditions hold ${ }^{13}$, the unique solution can be found using Karush-Kuhn-tucker (KKT) conditions. We first define the Lagrangian function of the problem,

$$
\begin{aligned}
\mathcal{L}(\boldsymbol{\phi}, \boldsymbol{\delta}, \boldsymbol{\gamma}, \eta)=\sum_{p=1}^{P} \frac{a_{p} \phi_{p}^{2}}{r_{p}^{\text {comp }}-\phi_{p}} & +\sum_{p=1}^{P}\left(\frac{1}{r_{p}^{\text {comp }}}+\frac{1}{r_{p}^{\text {comm }}}\right) \phi_{p} \\
& +\sum_{p=1}^{P} \delta_{p}\left(\underline{\phi}_{p}-\phi_{p}\right)+\sum_{p=1}^{P} \gamma_{p}\left(\phi_{p}-r_{p}^{\text {comp }}\right)-\eta\left(\sum_{p=1}^{P} \phi_{p}-1\right) .
\end{aligned}
$$

According to the KKT conditions, the optimal solution of the original optimization problem is a saddle point for the Lagrangian function (the first derivative of the Lagrangian function must be zero at the optimal point),

$$
\frac{a_{p}\left(r_{p}^{\mathrm{comp}}\right)^{2}}{\left(r_{p}^{\mathrm{comp}}-\phi_{p}\right)^{2}}+\xi_{p}-\delta_{p}+\gamma_{p}-\eta=0
$$

\footnotetext{
${ }^{13}$ Slater's conditions state the strong duality holds if there exists an strictly feasible point in the domain of the concave optimization problem. Thus, once can target the dual problem and use the Lagrangian method [37 Section 5.2.3].
} 
where $\xi_{p} \triangleq \frac{1}{r_{p}^{\text {comp }}}+\frac{1}{r_{p}^{\text {comm }}}-a_{p}$. It also satisfies the following feasibility conditions:

Primal feasibility: $\underline{\phi}_{p} \leq \phi_{p} \leq r_{p}^{\text {comp }}, \quad \sum_{p=1}^{P} \phi_{p}=1$,

Dual feasibility: $\delta_{p} \geq 0, \quad \gamma_{p} \geq 0$,

Complementary slackness: $\delta_{p}\left(\underline{\phi}_{p}-\phi_{p}\right)=0$,

$$
\gamma_{p}\left(\phi_{p}-r_{p}^{\text {comp }}\right)=0 .
$$

First, we note that $\gamma_{p}=0$, since $\gamma_{p} \neq 0$ makes $\phi_{p}=r_{p}^{\text {comp }}$, which cannot be a valid solution for (5). We study the cases when $\delta_{p}>0$ and $\delta_{p}=0$, separately:

[Case 1: Workers with $\delta_{p}>0$ ] Then, $\phi_{p}=\underline{\phi}_{p}$ and because of [5,

$$
\delta_{p}=\delta_{p}(\eta)=\frac{a_{p}\left(r_{p}^{\text {comp }}\right)^{2}}{\left(r_{p}^{\text {comp }}-\underline{\phi}_{p}\right)^{2}}+\xi_{p}-\eta,
$$

which must be positive requiring,

$$
\eta<\frac{a_{p}\left(r_{p}^{\text {comp }}\right)^{2}}{\left(r_{p}^{\text {comp }}-\underline{\phi}_{p}\right)^{2}}+\xi_{p} .
$$

[Case 2: Workers with $\delta_{p}=0$ ] Then (5) simplifies to,

$$
\begin{gathered}
\frac{a_{p}\left(r_{p}^{\text {comp }}\right)^{2}}{\left(r_{p}^{\text {comp }}-\phi_{p}\right)^{2}}+\xi_{p}-\eta=0, \\
\left(r_{p}^{\text {comp }}-\phi_{p}\right)^{2}=\frac{a_{p}\left(r_{p}^{\text {comp }}\right)^{2}}{\eta-\xi_{p}} .
\end{gathered}
$$

We note that (6) is only feasible when $\eta>\xi_{p}$. In this case, the valid solution for (6) is,

$$
\phi_{p}=r_{p}^{\text {comp }}\left(1-\sqrt{\frac{a_{p}}{\eta-\xi_{p}}}\right) .
$$

The other solution $\phi_{p}=r_{p}^{\text {comp }}\left(1+\sqrt{\frac{a_{p}}{\eta-x i_{p}}}\right)$ does not satisfy the constraint $\phi_{p} \leq r_{p}^{\text {comp }}$ and is invalid. The constraint $\phi_{p} \leq \underline{\phi}_{p}$ requires,

$$
\begin{aligned}
& r_{p}^{\text {comp }}\left(1-\sqrt{\frac{a_{p}}{\eta-\xi_{p}}}\right) \geq \underline{\phi}_{p} \\
& \frac{a_{p}}{\eta-\xi_{p}} \leq \frac{\left(r_{p}^{\text {comp }}-\phi_{p}\right)^{2}}{\left(r_{p}^{\text {comp }}\right)^{2}}
\end{aligned}
$$




$$
\eta \geq \frac{a_{p}\left(r_{p}^{\mathrm{comp}}\right)^{2}}{\left(r_{p}^{\mathrm{comp}}-\underline{\phi}_{p}\right)^{2}}+\xi_{p}
$$

which is a stronger inequality than $\xi_{p}>\eta$. Finally, we consider both cases together,

$$
\phi_{p}= \begin{cases}r_{p}^{\text {comp }}\left(1-\sqrt{\frac{a_{p}}{\eta-\xi_{p}}}\right), & \frac{a_{p}\left(r_{p}^{\text {comp }}\right)^{2}}{\left(r_{p}^{\text {comp }}-\underline{\phi}_{p}\right)^{2}}+\xi_{p} \leq \eta \\ \underline{\phi}_{p}, & \text { otherwise. }\end{cases}
$$

To incorporate the last remaining constraint, $\eta$ is set such that $\sum_{p=1}^{P} \phi_{p}=1$. Since the problem has a unique solution, there exists a unique $\eta$ such that above holds. Conversely, with the above choice, the system of KKT conditions holds with $\gamma_{p}=0, \eta$ such that $\sum_{p=1}^{P} \phi_{p}=1$, and $\delta_{p}=\max \left\{\delta_{p}(\eta), 0\right\}$, and this concludes the proof.

\section{APPENDIX C}

\section{ProOF OF THEOREM 2}

According to Theorem 1, and when $a_{p}=1 / r_{p}^{\text {comp }}$ and $r_{p}^{\text {comm }} \rightarrow \infty$, the optimal load split is,

$$
\phi_{p}=\max \left\{r_{p}^{\text {comp }}\left(1-\sqrt{\frac{1}{\eta r_{p}^{\text {comp }}}}\right), \underline{\phi}_{p}\right\}= \begin{cases}r_{p}^{\text {comp }}\left(1-\sqrt{\frac{1}{\eta r_{p}^{\text {comp }}}}\right), & \frac{r_{p}^{\text {comp }}}{\left(r_{p}^{\text {comp }}-\underline{\phi}_{p}\right)^{2}}<\eta \\ \underline{\phi}_{p}, & \text { otherwise. }\end{cases}
$$

Since the function $\frac{r}{\left(r-\underline{\phi}_{p}\right)^{2}}$ is strictly decreasing in $r>\underline{\phi}_{p}$ and the workers are sorted such that $r_{1}^{\text {comp }} \geq r_{2}^{\text {comp }} \geq \cdots \geq r_{P}^{\text {comp }}$

$$
\phi_{p}= \begin{cases}r_{p}^{\text {comp }}\left(1-\sqrt{\frac{1}{\eta_{p}^{\text {comp }}}}\right), & p \leq p^{*} \\ \underline{\phi}_{p} & \text { otherwise }\end{cases}
$$

Finally, for having $\sum_{p=1}^{P} \phi_{p}=1, \eta=\eta\left(p^{*}\right)$ must be a valid choice, i.e,

$$
\frac{r_{p^{*}}^{\text {comp }}}{\left(r_{p^{*}}^{\text {comp }}-\underline{\phi}_{p}\right)^{2}}<\eta\left(p^{*}\right) \leq \frac{r_{p^{*}+1}^{\text {comp }}}{\left(r_{p^{*}+1}^{\text {comp }}-\underline{\phi}_{p}\right)^{2}}
$$

Since the optimization problem has a unique solution, such unique $p^{*}$ exists.

\section{REFERENCES}

[1] G. E. Moore et al., "Cramming more components onto integrated circuits," 1965.

[2] G. Moore, "Moore's law," Electronics Magazine, vol. 38, no. 8, p. 114, 1965.

[3] S. Dutta, V. Cadambe, and P. Grover, "“'Short-Dot”: Computing large linear transforms distributedly using coded short dot products," IEEE Transactions on Information Theory, vol. 65, no. 10, pp. 6171-6193, 2019.

[4] A. Mallick, M. Chaudhari, U. Sheth, G. Palanikumar, and G. Joshi, "Rateless codes for near-perfect load balancing in distributed matrix-vector multiplication," Proceedings of the ACM on Measurement and Analysis of Computing Systems, vol. 3, no. 3, pp. 1-40, 2019. 
[5] Q. Yu, S. Li, N. Raviv, S. M. M. Kalan, M. Soltanolkotabi, and S. A. Avestimehr, "Lagrange coded computing: Optimal design for resiliency, security, and privacy," in The 22nd International Conference on Artificial Intelligence and Statistics. PMLR, 2019, pp. 1215-1225.

[6] J. Dean and L. A. Barroso, “The tail at scale," Communications of the ACM, vol. 56, no. 2, pp. 74-80, 2013.

[7] G. Joshi, E. Soljanin, and G. Wornell, "Efficient redundancy techniques for latency reduction in cloud systems," $A C M$ Transactions on Modeling and Performance Evaluation of Computing Systems (TOMPECS), vol. 2, no. 2, p. 12 , 2017.

[8] A. Ramamoorthy, A. B. Das, and L. Tang, "Straggler-resistant distributed matrix computation via coding theory: Removing a bottleneck in large-scale data processing," IEEE Signal Processing Magazine, vol. 37, no. 3, pp. 136-145, 2020.

[9] U. Sheth, S. Dutta, M. Chaudhari, H. Jeong, Y. Yang, J. Kohonen, T. Roos, and P. Grover, "An application of storageoptimal matdot codes for coded matrix multiplication: Fast k-nearest neighbors estimation," in 2018 IEEE International Conference on Big Data (Big Data). IEEE, 2018, pp. 1113-1120.

[10] R. G. D'Oliveira, S. El Rouayheb, and D. Karpuk, "GASP codes for secure distributed matrix multiplication," IEEE Transactions on Information Theory, vol. 66, no. 7, pp. 4038-4050, 2020.

[11] O. A. Wahab, A. Mourad, H. Otrok, and T. Taleb, "Federated machine learning: Survey, multi-level classification, desirable criteria and future directions in communication and networking systems."

[12] Q. Yu, M. A. Maddah-Ali, and S. Avestimehr, "Polynomial codes: an optimal design for high-dimensional coded matrix multiplication," in NIPS, 2017.

[13] N. Raviv, I. Tamo, R. Tandon, and A. G. Dimakis, "Gradient coding from cyclic MDS codes and expander graphs," IEEE Transactions on Information Theory, vol. 66, no. 12, pp. 7475-7489, 2020.

[14] Y. Sarikaya and O. Ercetin, "Motivating workers in federated learning: A stackelberg game perspective," IEEE Networking Letters, vol. 2, no. 1, pp. 23-27, 2019.

[15] W. Y. B. Lim, N. C. Luong, D. T. Hoang, Y. Jiao, Y.-C. Liang, Q. Yang, D. Niyato, and C. Miao, "Federated learning in mobile edge networks: A comprehensive survey," IEEE Communications Surveys \& Tutorials, vol. 22, no. 3, pp. 2031-2063, 2020.

[16] S. Feng, D. Niyato, P. Wang, D. I. Kim, and Y.-C. Liang, "Joint service pricing and cooperative relay communication for federated learning," in 2019 International Conference on Internet of Things (iThings) and IEEE Green Computing and Communications (GreenCom) and IEEE Cyber, Physical and Social Computing (CPSCom) and IEEE Smart Data (SmartData). IEEE, 2019, pp. 815-820.

[17] D. Malak, A. Cohen, and M. Médard, "How to distribute computation in networks," in IEEE INFOCOM 2020 - IEEE Conference on Computer Communications, 2020, pp. 327-336.

[18] K. R. Duffy and S. Shneer, "MDS coding is better than replication for job completion times," Operations Research Letters, vol. 49, no. 1, pp. 91-95, 2021.

[19] S. Li, M. A. Maddah-Ali, and A. S. Avestimehr, "A unified coding framework for distributed computing with straggling servers," in 2016 IEEE Globecom Workshops (GC Wkshs). IEEE, 2016, pp. 1-6.

[20] K. Lee, C. Suh, and K. Ramchandran, "High-dimensional coded matrix multiplication," in 2017 IEEE International Symposium on Information Theory (ISIT). IEEE, 2017, pp. 2418-2422.

[21] M. Kim, J.-y. Sohn, and J. Moon, "Coded matrix multiplication on a group-based model," in 2019 IEEE International Symposium on Information Theory (ISIT). IEEE, 2019, pp. 722-726.

[22] K. Lee, M. Lam, R. Pedarsani, D. Papailiopoulos, and K. Ramchandran, "Speeding up distributed machine learning using codes," IEEE Transactions on Information Theory, vol. 64, no. 3, pp. 1514-1529, 2017.

[23] R. Tandon, Q. Lei, A. G. Dimakis, and N. Karampatziakis, "Gradient coding," arXiv preprint arXiv:1612.03301, 2016.

[24] — " "Gradient coding: Avoiding stragglers in distributed learning," in International Conference on Machine Learning. PMLR, 2017, pp. 3368-3376.

[25] N. Raviv, R. Tandon, A. Dimakis, and I. Tamo, "Gradient coding from cyclic MDS codes and expander graphs," in International Conference on Machine Learning. PMLR, 2018, pp. 4305-4313.

[26] S. Dutta, M. Fahim, F. Haddadpour, H. Jeong, V. Cadambe, and P. Grover, "On the optimal recovery threshold of coded matrix multiplication," IEEE Transactions on Information Theory, vol. 66, no. 1, pp. 278-301, 2020.

[27] Q. Yu, M. A. Maddah-Ali, and A. S. Avestimehr, "Polynomial codes: an optimal design for high-dimensional coded matrix multiplication," arXiv preprint arXiv:1705.10464, 2017. 
[28] T. Baharav, K. Lee, O. Ocal, and K. Ramchandran, "Straggler-proofing massive-scale distributed matrix multiplication with d-dimensional product codes," in 2018 IEEE International Symposium on Information Theory (ISIT). IEEE, 2018, pp. 1993-1997.

[29] S. Li, M. A. Maddah-Ali, and A. S. Avestimehr, "Coded mapreduce," in 2015 53rd Annual Allerton Conference on Communication, Control, and Computing (Allerton). IEEE, 2015, pp. 964-971.

[30] M. A. Attia and R. Tandon, "Near optimal coded data shuffling for distributed learning," IEEE Transactions on Information Theory, vol. 65, no. 11, pp. 7325-7349, 2019.

[31] L. Song, C. Fragouli, and T. Zhao, "A pliable index coding approach to data shuffling," IEEE Transactions on Information Theory, vol. 66, no. 3, pp. 1333-1353, 2019.

[32] L. Kleinrock, Queuing Systems Vol. I: Theory. New York: Wiley, 1975.

[33] T. Baharav, K. Lee, O. Ocal, and K. Ramchandran, "Straggler-proofing massive-scale distributed matrix multiplication with d-dimensional product codes," in IEEE International Symposium on Information Theory (ISIT), 2018, pp. $1993-1997$.

[34] R. G. Gallager, Stochastic processes: theory for applications. Cambridge University Press, 2013.

[35] J. L. Bentley, "Multidimensional binary search trees used for associative searching," Communications of the ACM, vol. 18, no. 9, pp. 509-517, 1975.

[36] R. Nowak, "Generalized binary search," in 2008 46th Annual Allerton Conference on Communication, Control, and Computing. IEEE, 2008, pp. 568-574.

[37] S. Boyd, S. P. Boyd, and L. Vandenberghe, Convex optimization. Cambridge university press, 2004. 